\title{
Spatial structure of the geodesic acoustic mode in the FT-2 tokamak by Upper Hybrid Resonance Doppler Backscattering
}

\author{
A D Gurchenko ${ }^{1,2}$, E Z Gusakov ${ }^{1,2}$, A B Altukhov ${ }^{1,2}$, E P Selyunin ${ }^{1,2}$, \\ L A Esipov ${ }^{1,2}$, M Yu Kantor ${ }^{1,2}$, D V Kouprienko ${ }^{1,2}$, S I Lashkul ${ }^{1,2}$, \\ A Yu Stepanov ${ }^{1,2}$ and $F$ Wagner ${ }^{3,2}$ \\ ${ }^{1}$ Ioffe Institute, St. Petersburg, Russia \\ ${ }^{2}$ St. Petersburg State Polytechnical University, RLPAT, St. Petersburg, Russia \\ ${ }^{3}$ Max-Planck-Institut für Plasmaphysik, EURATOM Association, Greifswald, Germany
}

\begin{abstract}
A new experimental technique is presented for the study of zonal flows in tokamak plasmas - Upper Hybrid Resonance Doppler Backscattering. The feasibility of the diagnostics is demonstrated allowing a complete characterisation of the temporal and spatial behaviour of geodesic acoustic modes. The experimental results are obtained at the FT-2 tokamak.
\end{abstract}

\section{Introduction}

Geodesic acoustic modes (GAMs) manifesting themselves in toroidal plasmas as symmetric oscillations of the electrostatic potential (with poloidal and toroidal mode numbers $m=n=0$ ) coupled with a small density perturbation (with $\mathrm{m}=1, \mathrm{n}=0$ ) and low-frequency zonal flows are considered nowadays as features of micro-turbulence induced self-control [1]. According to analytical results and numerical modelling this control occurs in two ways. Firstly, GAMs are driven by the turbulent Reynolds stress in an energy conserving three-wave-interaction process so that their generation necessarily corresponds to a reduction of drift-wave energy. Secondly, GAMs suppress long-scale fluctuations by shearing of the drift-wave eddies and participate in nonlinear transfer of energy to smaller radial scales where it is linearly dissipated.

Experimental information on GAMs in high temperature plasma is usually obtained either via direct measurement of the plasma potential by heavy-ion-beam probe (HIBP) technique, or via measurements of the oscillating component of the micro-turbulence rotation velocity by beam emission spectroscopy (BES) or Doppler reflectometry, whereas at the plasma edge GAMs are diagnosed by Langmuir probes [2]. Though rather detailed information is obtained from these techniques, they are limited, however, to centimetre range resolution causing interpretation problems for the GAM spatial structure, its propagation and correlation properties [3-6]. 


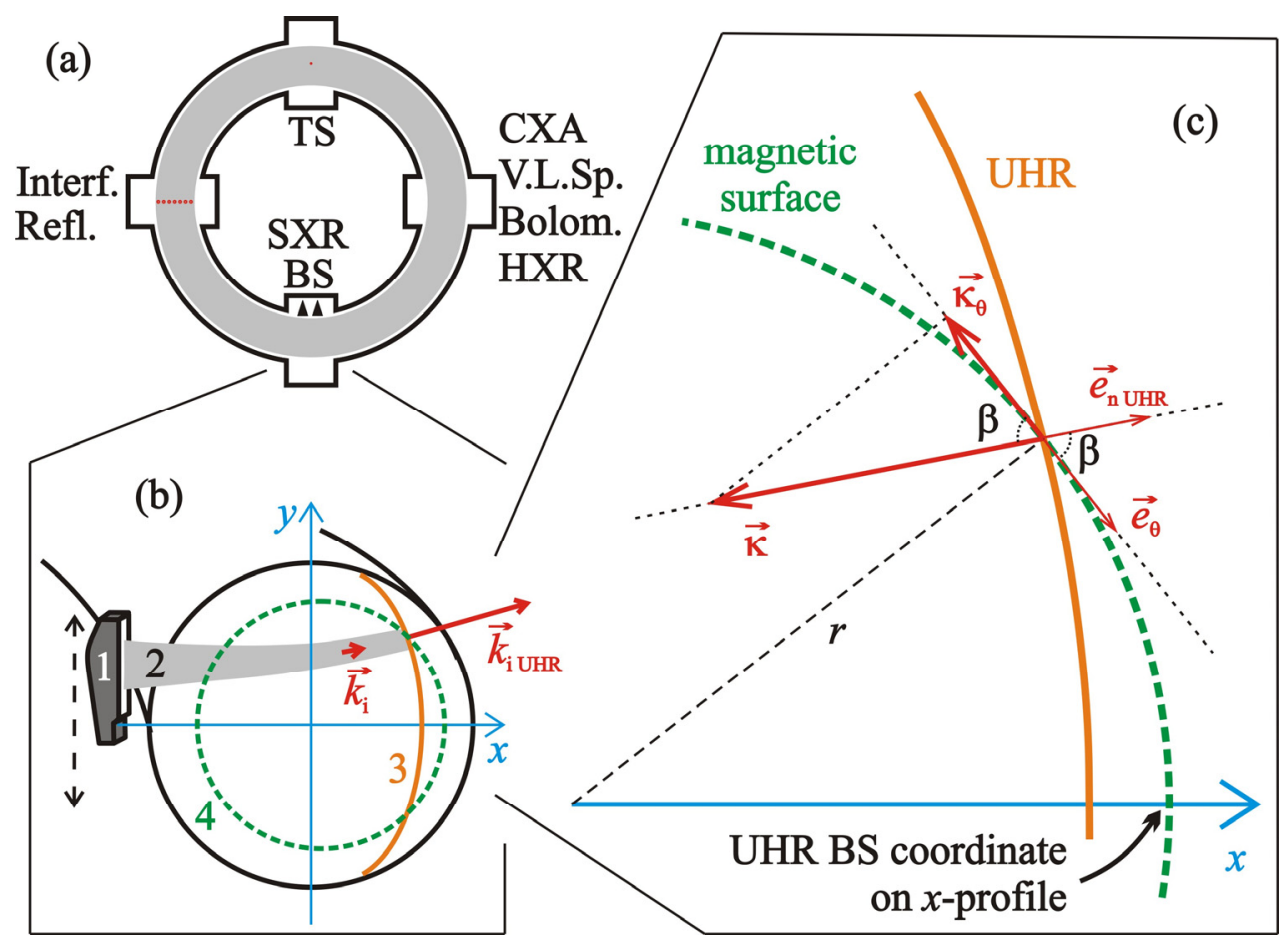

Figure 1. (a) Tokamak FT-2 top view (diagnostics' arrangement: BS - UHR BS; SXR - soft $X$-ray detectors; Interf. - 7 channel 2-mm interferometer; Refl. - $O$-mode reflectometer; TS - multi-pass intracavity Thomson scattering; CXA - 5 channel charge exchange analyzer; V.L.Sp. - visible light spectroscopy; Bolom. - bolometer; HXR - hard $X$-ray detector). (b) Poloidal cross-section with the UHR BS diagnostic $(1-X$-mode moveable double antenna set; 2 - probing beam; 3 - the UHR surface; 4 the magnetic surface). The point $x=0, y=0$ corresponds to the geometrical center of the chamber. $(c)$ The geometry of fluctuation's wave vector relative to the UHR and magnetic surfaces. (Colours adapted to grey scale in the printed version of the article.)

In the present paper we introduce a new microwave technique - the upper hybrid resonance (UHR) Doppler backscattering (BS), offering superior spatial resolution and we demonstrate its feasibility for diagnosing the GAM temporal and spatial characteristics.

The paper consists of five sections. After introduction and brief description of the UHR Doppler BS technique (UHR BS) in the first two sections, the investigation of the oscillating behaviour of plasma poloidal rotation is presented in section 3, compiling clear evidence that indeed GAMs are observed in the FT-2 tokamak. The complete data set on GAM characteristics and the radial distribution of major parameters are obtained in low (19 kA) current linear ohmic regime with hydrogen as the main gas under quasi-stationary conditions. The isotope effect on the GAM frequency is demonstrated in deuterium discharges. Modification of GAM localisation at higher ( $32 \mathrm{kA}$ ) current due to growth of its damping and possibility of GAM observation at the background of strong MHD activity in dynamical regime with fast current ramp up (CRU) is demonstrated.

The GAM spatial characteristics (radial correlation length and wave number) are obtained from two microwave scattering techniques (one based exclusively on UHR BS and the second one utilizing a microwave reflectometry channel) and are compared in section 4 . The possibility of UHR BS to study the three-wave-interaction of the GAM with broadband smallscale turbulence is demonstrated in section 5 . 


\section{The UHR Doppler backscattering diagnostics}

The UHR BS technique [7,8] utilizes $X$-mode microwave plasma probing out of the tokamak's equatorial plane from the high magnetic field side as shown in figure 1. The diagnostic is benefiting from the growth of electric field and wave numbers in the UHR resulting in enhanced scattering signal, sub-mm radial resolution and substantially increased Doppler frequency shift $\left(f_{\mathrm{D}}\right)$. The absolute value of the wave vector in the UHR $\vec{k}_{\mathrm{i} U \mathrm{HR}}$ is usually by an order of magnitude larger than its value on the way to the UHR $\vec{k}_{\mathrm{i}}$ (as it is shown in figure 1(b) by arrows with different lengths). The diagnostic poloidal resolution depends on the width of the probing beam in the UHR. Due to the non-equatorial probing, the wave vector, which is nearly normal to the UHR surface $\vec{k}_{\mathrm{iUHR}} \cong\left|\vec{k}_{\mathrm{i} U H R}\right| \vec{e}_{\mathrm{n} U H R}$, has significant projections both onto radial $(r)$ and poloidal $(\theta)$ directions. The fluctuation wave vector leading to BS in the UHR ( $\vec{\kappa}$ ) due to the Bragg condition has also a component tangential to the magnetic surface (see figure 1(c)) $\kappa_{\theta}=\kappa \cos \beta=\kappa\left(\vec{e}_{\mathrm{nUHR}} \cdot \vec{e}_{\theta}\right)$, which is usually larger than the absolute value of the total wave vector $\vec{k}_{\mathrm{i}^{*}}$ at a distance of a few millimetres from the UHR. This effect increases the frequency shift $\left(f_{\mathrm{D}}\right)$ of the UHR BS frequency spectrum:

$$
2 \pi f_{\mathrm{D}}=2 k_{\mathrm{iUHR} \theta} V_{\theta}=\kappa_{\theta} V_{\theta},
$$

proportional to the poloidal phase velocity of the fluctuation $\left(V_{\theta}\right)$. The frequency shift $(1)$ is composed of the drift wave frequency and the Doppler frequency shift associated with plasma poloidal rotation. The former one according to the theoretical predictions [9] and recent experimental results [10] is small in the case of fine-scale fluctuations. Employing correlation techniques to the Doppler UHR BS method [7] allows to determine the $\kappa$ value and finally to reconstruct the poloidal wave phase velocity from the frequency shift measurements.

\section{GAM observation and characterization}

\subsection{Parameters of the FT-2 tokamak hydrogen low-current discharge}

The main set of measurements was carried out in FT-2 tokamak [11] $(R=55 \mathrm{~cm} ; a=7.9 \mathrm{~cm})$ hydrogen discharges with low plasma current $I_{\mathrm{p}} \sim 19 \mathrm{kA}$ and a toroidal magnetic field $B_{\mathrm{T}} \sim 2.2 \mathrm{~T}$. The discharge parameters are as follows: central density $n_{\mathrm{e} 0} \sim 4.5 \times 10^{19} \mathrm{~m}^{-3}$, central electron $T_{\mathrm{e} 0} \sim 470 \mathrm{eV}$ and ion $T_{\mathrm{i} 0} \sim 110 \mathrm{eV}$ temperatures, effective charge number $Z_{\mathrm{eff}} \sim 3.5$ with oxygen $\mathrm{O}^{8+}$, as a main impurity. The basic discharge waveforms are shown in figure 2(ad). The corresponding spatial profiles, measured during the quasi-stationary phase $(28-30 \mathrm{~ms})$ of the shot possessing a total duration of $60 \mathrm{~ms}$, are shown in figure 2(e-h) together with ASTRA code reconstruction for the safety factor $q$. Profiles are presented in their dependence on the equatorial $x$-coordinate, which is introduced in figure $1(\mathrm{~b}),(\mathrm{c})$. The arrangement of diagnostics measuring different experimental parameters is shown in figure 1(a). The width of curves in figure 2(e-g) corresponds to experimental error bars. The UHR surface's location is at $r / a \sim 0.4-1$ for a probing frequency $f_{\mathrm{i}} \sim 55-68 \mathrm{GHz}$. 


\subsection{Experimental approach for the investigation of the poloidal rotation dynamics}

The method of poloidal rotation dynamics study with UHR BS utilized in the present paper is, in essence, similar to that proposed in [12] for Doppler reflectometry. The quadrature (i.e. with in-phase and quadrature (IQ) signals' detection) UHR BS scheme introduced in [13] was used for this purpose in the experiments with minor changes. The moveable focusing double $X$-mode antennae set at the high field side (see figure 1(b)) was positioned vertically at $y=+15 \mathrm{~mm}$ above the equatorial plane for Doppler BS measurements. The pair of sine-cosine (IQ) homodyne signals at frequencies of the turbulent fluctuations was used for reconstruction of the bilateral spectrum equivalent to the heterodyne BS spectrum. The data acquisition
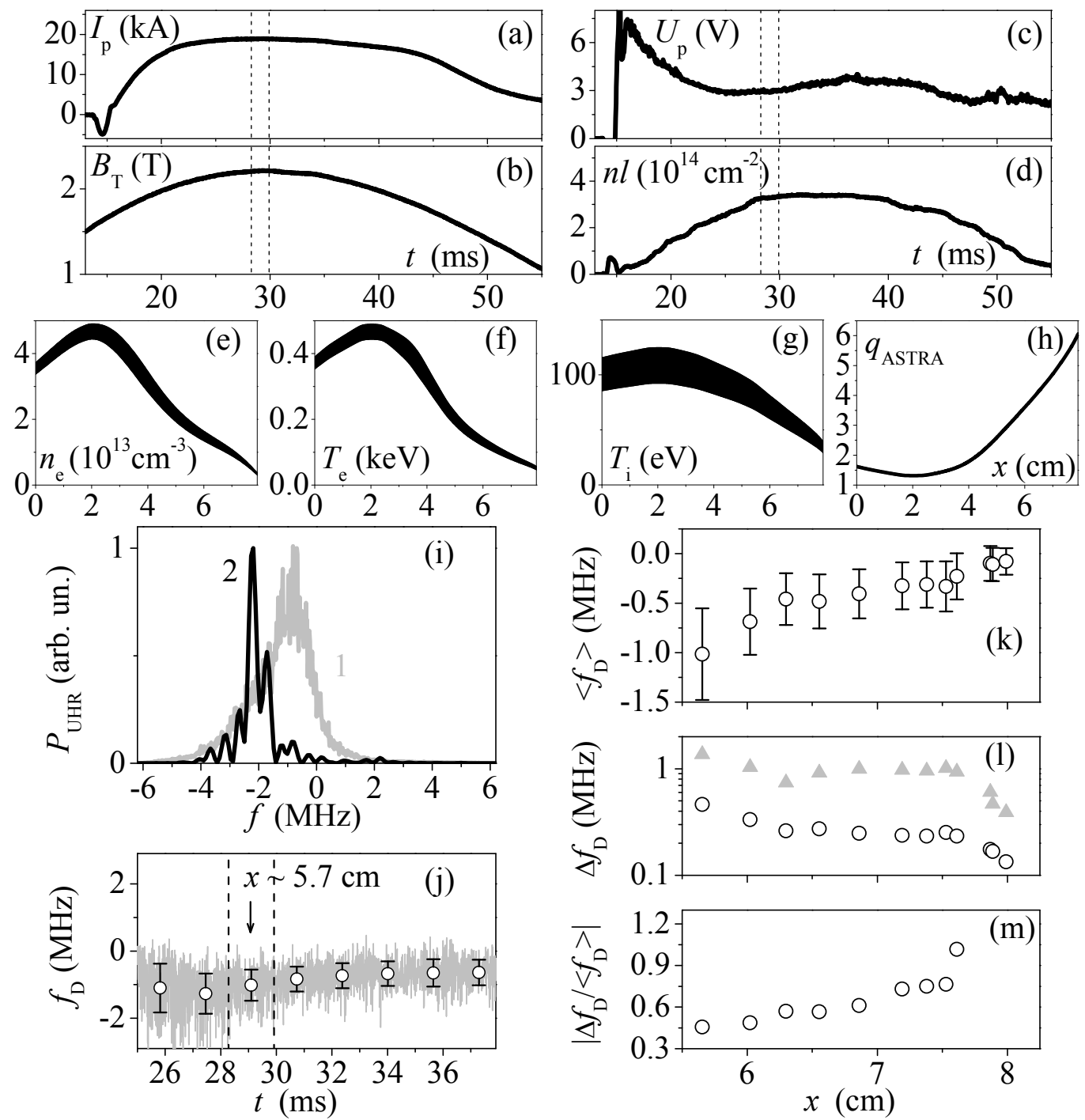

Figure 2. The 19 kA hydrogen regime. Evolutions of the $(a)$ plasma current; $(b)$ toroidal magnetic field; (c) loop voltage; $(d)$ averaged central density. $(e)$ The density profile along the equatorial plane; $(f)$ electron and $(g)$ ion temperature profiles; $(h)$ the safety factor profile. $(i)$ The UHR BS spectra measured at $x \sim 5.7 \mathrm{~cm}(1-$ calculated with averaging over 63 realizations; 2 - calculated in narrow window without averaging). ( $j$ ) The time trace of the Doppler frequency shift for $f_{\mathrm{i}} \sim 66 \mathrm{GHz}$. Profiles of the $(k)$ $f_{\mathrm{D}}$ mean value; $(l) f_{\mathrm{D}}$ rms value (circles) and the width of the $P_{\mathrm{UHR}}$ spectrum at the half of amplitude (triangles); $(m)$ normalized $f_{\mathrm{D}}$ rms value. 
frequency for BS signals in different cases considered in this paper was 20-50 MHz. An example of the UHR BS spectrum averaged over $1.6 \mathrm{~ms}$ is shown in figure 2(i) by the grey curve 1 , where $f=f_{\mathrm{s}}-f_{\mathrm{i}}$ corresponds to the frequency shift (1), $f_{\mathrm{i}}=66 \mathrm{GHz}$ and $f_{\mathrm{s}}$ is the scattering signal frequency. The sampling rate in this case was $20 \mathrm{MHz}$. The window with 1024 data points was used in one FFT realization. Each next window was started with a shift of 512 points from the previous window beginning. The averaged spectrum was calculated over 63 realizations. A negative and positive sign of $f$ indicates the fluctuation phase velocity direction correspondingly along and opposite to the electron diamagnetic drift direction. The spectrum is wide and has a significant Doppler frequency shift $f_{\mathrm{D}} \sim 800 \mathrm{kHz}$ in the electron diamagnetic drift direction (see grey curve 1 in figure 2(i)). However when calculated without averaging in $3.2 \mu$ s window (the window with 64 data points was used for single FFT realization) the power spectrum $\left(P_{\mathrm{UHR}}\right)$ appears to be narrow and possesses a frequency shift different from the averaged one given by curve 1 (see black curve 2 in figure 2(i)). The Doppler frequency shift $f_{\mathrm{D}}$ temporal behaviour estimated with $3.2 \mu$ s sampling interval from the variation of the non-averaged spectra as:

$$
f_{\mathrm{D}}(t)=\frac{\int f\left|P_{\mathrm{UHR}}(f, t)\right| d f}{\int\left|P_{\mathrm{UHR}}(f, t)\right| d f},
$$

is presented in figure 2(j) by the grey curve, whereas the mean values determined in $1.6 \mathrm{~ms}$ windows are shown by open circles. Integration limits in the above formula (2) should be chosen such as to exclude an influence of asymmetrical spectral wings on the calculation of the spectrum's center of gravity. In the case presented in figure 2 the integration interval was determined as the half of amplitude level of the spectrum $P_{\text {UHR }}$. Typical features of the $f_{\mathrm{D}}(t)$ dependence are comparable values of its mean value $\left\langle f_{\mathrm{D}}>\right.$ and rms value $\Delta f_{\mathrm{D}}$ (shown by black error bars in figure $2(\mathrm{j}))$.

Spatial profiles of the $\left\langle f_{\mathrm{D}}>, \Delta f_{\mathrm{D}}\right.$ and the ratio of rms to mean value $\Delta f_{\mathrm{D}} /\left\langle f_{\mathrm{D}}>\right.$ at $t \sim 29 \mathrm{~ms}$ are shown in figure $2(\mathrm{k}-\mathrm{m})$ by open circles. The $x$ coordinate here corresponds to the cross-section of the magnetic surface intersecting the UHR BS position with the major radius axis in the equatorial plane $x$-axis (see figure $1(\mathrm{c})$ )). The $\Delta f_{\mathrm{D}}$ profile is compared with the spatial distribution of the averaged spectrum's width at half of the amplitude (see grey triangles in figure 2(1)). Both dependencies are plotted in logarithmic scale and look similar. Poloidal wave numbers of fluctuations $\kappa_{\theta}$ leading to the UHR BS, extracted from the radial correlation measurements $[7,8]$, make it possible to estimate the poloidal phase velocity $V_{\theta}$ and the radial electric field $E_{r}$ from $\left\langle f_{\mathrm{D}}>\right.$ values. The experimental data obtained in this regime were compared to results of the FT-2 tokamak gyro-kinetic modelling using the ELMFIRE code $[14,10]$. The spatial distributions of the poloidal $E_{r} \times B$ velocity and the radial electric field, both their mean and rms values and even the $E_{r}$ time trace were found to be in good agreement with those measured experimentally. It should be underlined that the amplitude (rms) of electric field oscillations observed at FT-2 (see figure 2(m)) is comparable to its mean value. Due to substantial inhomogeneity of these oscillations, shown in section 4 of this paper, they could lead to strong shearing of the plasma poloidal flow influencing small-scale drift wave turbulence.

\subsection{Spectral characteristics of the $f_{\mathrm{D}}(t)$ signal}

The Fourier spectrum of the $f_{\mathrm{D}}(t)$ dependence, calculated with averaging over 8 realizations (with FFT windows taken one by one) within an $1.6 \mathrm{~ms}$ interval between the dashed lines in figure 2(j) is presented in figure 3(a) by black curve 1. An intensive spectral line is clearly 
seen at $F \sim 54 \pm 1.2 \mathrm{kHz}$. The grey solid line 2 corresponds to the mean noise level, which can be attributed to perturbations of radial electric field $E_{r}$ and density, as well as distortions of the UHR and magnetic surfaces. The ratio of the line's amplitude $P_{\max } \sim 5.41 \pm 0.03$ to the noise level $P_{\mathrm{n}} \sim 1.55 \pm 0.47$ at peak's frequency is a contrast $C=P_{\max } / P_{\mathrm{n}} \sim 3.5 \pm 1.1$. The contrast characterizing the signal-to-noise ratio indicate reliability of the line observation. The spatial scan of the $f_{\mathrm{D}}(t)$ spectrum yields the radial dependence of the line frequency. The radial profiles of the spectral line's contrast $C$ and frequency $F$ at $t \sim 29 \mathrm{~ms}$ are shown in figure $3(\mathrm{~b}),(\mathrm{c})$ by circles. The mean value of the $f_{\mathrm{D}}(F)$ noise in the terms of contrast corresponds to the level $C=1$. The grey curve 1 in figure 3(b) is the rms value of this noise. It should be explained that the line frequency $F$ was determined only for cases where the contrast error bars were not crossing the grey curve 1 in figure 3(b).

The theoretical estimations of the GAM frequency $\left(F_{\mathrm{G}}\right)$ accounting for spatial variation of plasma parameters are also plotted in figure $3(\mathrm{c})$. The grey curve 1 was obtained with formula:

$$
F_{\mathrm{G}}^{2} \sim \frac{1}{2 \pi^{2} R^{2} m_{\mathrm{i}}}\left(\frac{7}{4} T_{\mathrm{i}}+Z_{\mathrm{i}} T_{\mathrm{e}}\right),
$$

with $m_{\mathrm{i}}=m_{\mathrm{H}}$ (the ion hydrogen mass) and $Z_{\mathrm{i}}=1\left(\right.$ for $\left.\mathrm{H}^{+}\right)$. This formula was introduced in [15] where a numerical calculation of the time evolution of the axisymmetric potential was compared with the kinetic treatment [16]. It also follows from the kinetic analysis accounting for multiple ion species [17]. The first term in this formula was separately considered in [18] under condition $T_{\mathrm{i}} \gg>T_{\mathrm{e}}$ when it dominates. Values of the line's frequency are lying below the curve 1 but above the level provided by (3) at $T_{\mathrm{i}}=0$ (dashed grey curve 2) that is usual in experiment [2]. The black curve 3 , which provides the best fit to the experimental points, is based on the analytical prediction of the GAM frequency, obtained in [17] for plasmas with two ion species, when the $\mathrm{O}^{8+}$ impurity component was taken into account. This agreement permits the preliminarily conclusion that the dominant line in the $f_{\mathrm{D}}(F)$ spectrum results from the interaction with GAMs. It should be mentioned that the spatial distribution of the GAM frequency obtained from $E_{r}(t)$ dependence in ELMFIRE simulation $[14,10]$ was also very
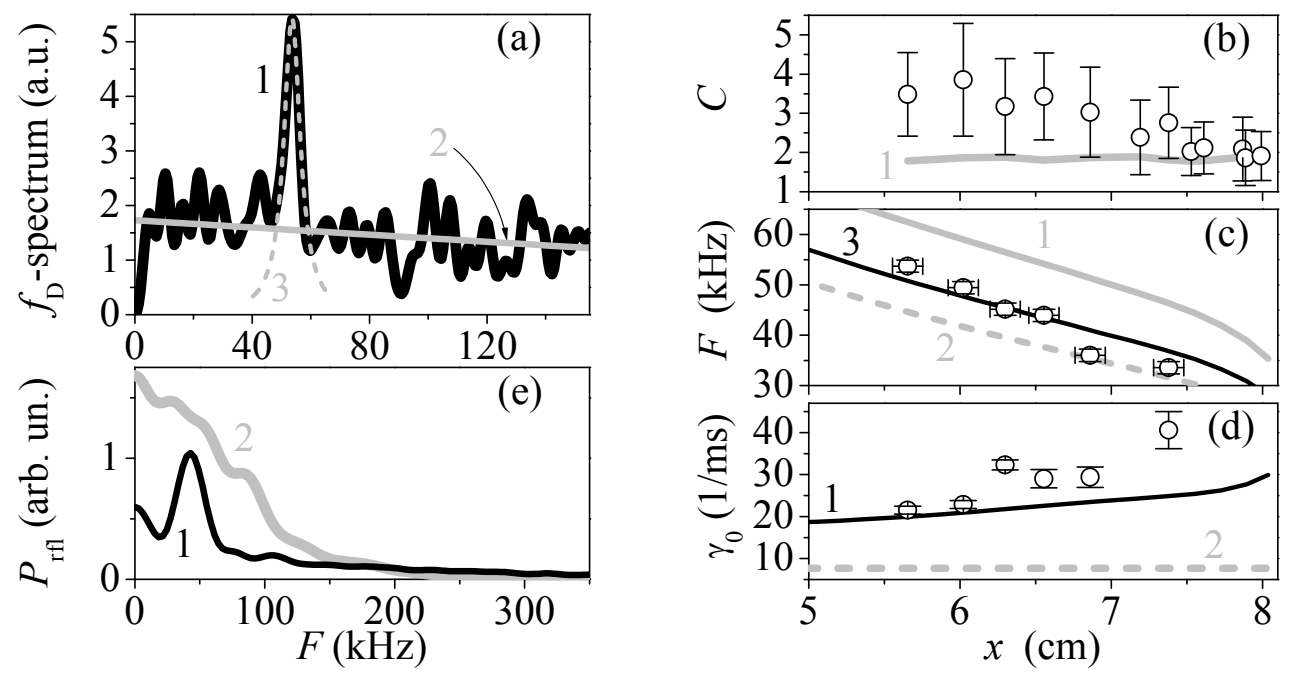

Figure 3. The $19 \mathrm{kA}$ hydrogen regime. (a) The spectrum of the Doppler frequency shift time trace. Radial profiles of the dominating line's $(b)$ contrast and $(c)$ frequency. $(d)$ The profile of the fitting parameter $\gamma_{0}$ chargeable for the spectral line's width (dashed grey line 2 corresponds to the $f_{\mathrm{D}}$-spectrum resolution $\pi \Delta F)$. (e) The homodyne $O$-mode reflectometric power spectrum for $f_{\mathrm{i}} \sim 28 \mathrm{GHz}(1-$ top launching, vertical probing; 2 - equatorial probing from low field side). 
close to the experimental dependence in figure 3(c).

The GAM spectral line in figure 3(a) is well approximated by a Lorenzian dependence (dashed grey curve 3 ) describing the excitation of a dissipative oscillator by a random force presumably provided here by Reynolds stress:

$$
P_{\mathrm{G}}=\frac{A_{\text {turb }}^{2}}{4 \pi^{2}\left(F-F_{0}\right)^{2}+\gamma_{0}^{2}},
$$

with the fitting parameters: $F_{0} \sim F_{\mathrm{G}}, \gamma_{0}=21.5 \pm 0.9[1 / \mathrm{ms}]$ and $A_{\text {turb }}$ proportional to the Reynolds stress Fourier component at frequency $F$. The radial dependence of $\gamma_{0}$ is shown by open circles in figure 3(d). It is compared there with the sum of collisional $\gamma_{\mathrm{c}} \sim 4 v_{\mathrm{i}} / 7$ and Landau $\gamma_{\mathrm{L}} \sim 2 \pi F_{\mathrm{G}} \exp \left(-q^{2}\right)$ damping rates (black curve 1) responsible for GAM damping in warm plasmas [2]. These damping rates were estimated with appropriate corrections according to the theory [17], in which the role of impurities $\left(Z_{\text {eff }} \sim 3.5\right)$ is accounted for. As it is seen in figure $3(\mathrm{~d}), \gamma_{0}$ is close to the theoretical prediction in the gradient zone and exceeds it by a factor of 2 at the edge, which is probably due to neglecting ion-neutral collisions significant in this region. It should be mentioned that the observed $\gamma_{0}$ growth is consistent with the decrease of the GAM line contrast seen at the edge in figure 3(b), which makes the line invisible against the background noise level for $x>7.5 \mathrm{~cm}$.

The GAM origin of the observed oscillations of plasma poloidal velocity was also confirmed by reflectometry diagnostics situated in the FT-2 cross-section shifted by 90 degrees in toroidal direction (see figure 1(a)). The homodyne detected reflectometry spectra obtained with top and equatorial launch are shown in figure 3(e). As it is seen, the line at the GAM frequency dominates in the case of top incidence of the probing power and is missing in the spectrum obtained with equatorial launching. This angular dependence agrees well with the property of the density perturbation associated with the GAM, which possessing $(m=1$, $n=0)$ symmetry is proportional to $\sin (\theta)$ and therefore should be negligible in the equatorial plane [2].

\subsection{GAM frequency dependence on the ion mass}

In order to additionally confirm the GAM origin of the dominating line in the $f_{\mathrm{D}}$-spectrum its frequency dependence on the ion mass was investigated in $19 \mathrm{kA}$ deuterium discharges. The main parameters were adapted so that the new regime was similar to the hydrogen $19 \mathrm{kA}$ one. The resulting experimental profiles are presented in figure 4(a-c) together with the safety factor reconstruction (figure 4(d)).

An example of the $f_{\mathrm{D}}(F)$ spectrum at $x \sim 5.5 \mathrm{~cm}$ in the $19 \mathrm{kA}$ deuterium regime is shown in figure 4(e). The contrast of the main peak of the spectrum is $C \sim 10.2 \pm 3.4$, which is higher than in the hydrogen. This difference, at least partly, could be explained based on expression 4 by a smaller value in deuterium of the GAM damping rate proportional to the ion collision frequency $\left(\gamma_{\mathrm{c}} \sim 4 v_{\mathrm{i}} / 7\right)$. The spatial profiles of the dominating spectral line's contrast $C(x)$ and frequency $F(x)$ are shown in figure 4(f), (g). As it is seen in figure 4(f), the GAM line contrast is growing toward the core of the discharge similar to the hydrogen case.

It is worth to note that in deuterium the GAM frequency is substantially lower $(F \sim 30-40 \mathrm{kHz}$ in the region $x \sim 5.5-6.5 \mathrm{~cm}$ ) than in hydrogen where it was $F \sim 45-55 \mathrm{kHz}$ (see figure 3(c)), which is in qualitative agreement with expression (3). The values of the line's frequency in figure $4(\mathrm{~g})$ are lying between estimations made with formula 3 (grey curves 1 and 2). However due to the high $Z_{\text {eff }}$ level in the FT-2 discharge $\left(Z_{\text {eff }} \sim 3.5\right)$ the quantitative agreement of the experimental data to theoretical predictions can be only obtained accounting for 

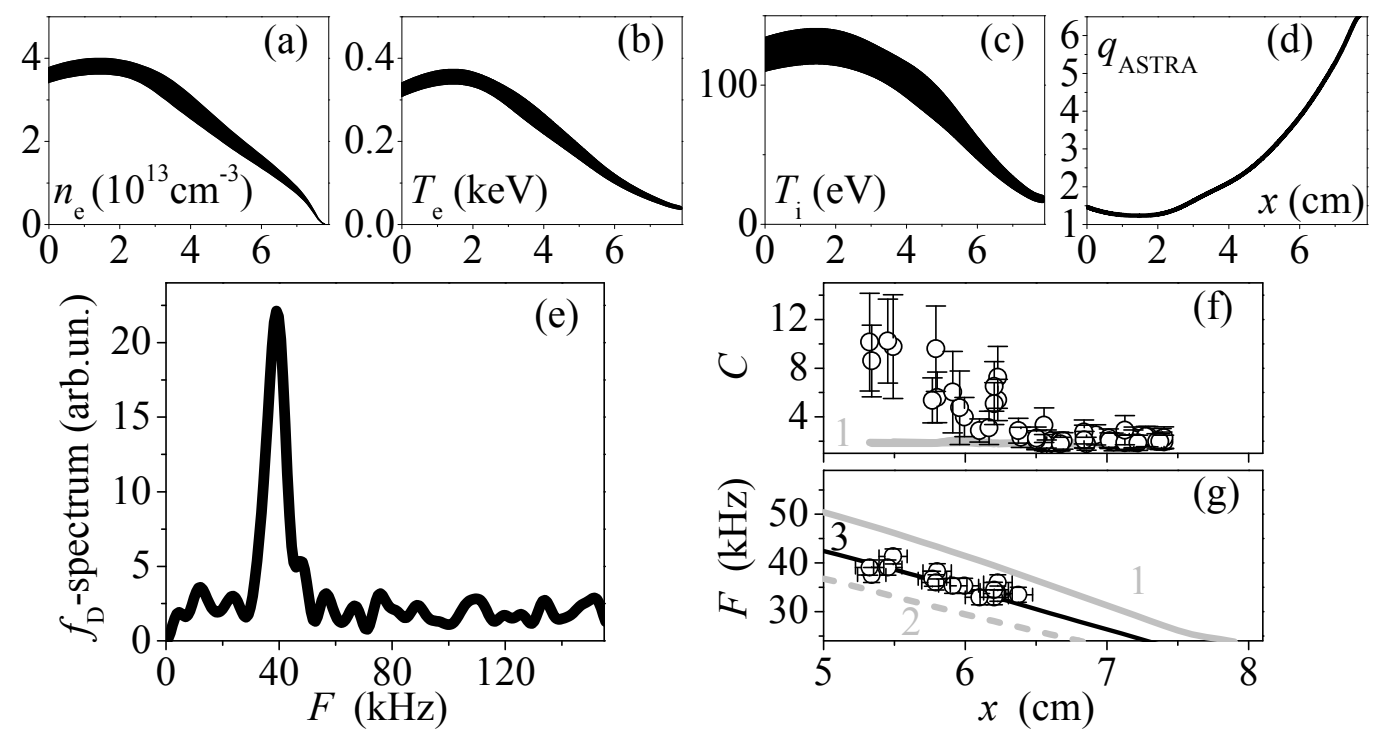

Figure 4. The $19 \mathrm{kA}$ deuterium regime. Experimental radial profiles of ( $a$ ) the density; $(b)$ electron and $(c)$ ion temperature. $(d)$ The safety factor profile. $(e)$ The $f_{\mathrm{D}}$ spectrum at $x \sim 5.5 \mathrm{~cm}$. Radial profiles of the dominating line's $(f)$ contrast and $(g)$ frequency.

impurities. The black curve 3, calculated with formula from ref. [17] for plasma with two ion species $\left(\mathrm{D}^{+}\right.$and $\mathrm{O}^{8+}$ in this case) is very close to the experimental points, as it was in the case of hydrogen.

\subsection{Observation of GAM in high current hydrogen discharge}

The interesting feature of the contrast profile $C(x)$ measured in the $19 \mathrm{kA}$ regime was its monotonous increase to smaller radii up to the inner accessibility boundary of the UHR BS measurements. This dependence could be explained by the GAM damping variation in the framework of a model of oscillator driven by a random force resulting in expression (4). The damping parameter, introduced as:

$$
D_{\gamma}(x)=\exp \left(-\left(\gamma_{\mathrm{L}}+\gamma_{\mathrm{c}}\right) \Delta t\right)
$$

where $\Delta t$ is taken equal to the averaged GAM period, is shown in figure 5(e) as grey curve 1. In the area of the UHR BS measurements $(x>5.5 \mathrm{~cm})$ due to extremely small level of Landau damping (in the range $x>5 \mathrm{~cm}: \gamma_{\mathrm{L}}<7 \times 10^{-3} 1 / \mathrm{ms}$ ) it is monotonously increasing towards the discharge centre indicating decreasing GAM damping in accordance with the $C(x)$ dependence.

In order to enhance the Landau damping in the observation region the plasma current was increased up to $I_{\mathrm{p}} \sim 32 \mathrm{kA}$. Experimental profiles of the main plasma parameters are presented in figure 5(a-c) together with ASTRA code reconstruction for the safety factor $q$ in figure 5(d) The main impurity was oxygen $\mathrm{O}^{8+}$ with $Z_{\text {eff }} \sim 3$. The new damping profile is shown in figure $5(\mathrm{e})$ by black curve 2 .

An example of the $f_{\mathrm{D}}(F)$ spectrum at $x \sim 6.2 \mathrm{~cm}$ in the $32 \mathrm{kA}$ regime is shown in figure 5(f). The contrast of the main peak of the spectrum is $C \sim 7.4 \pm 2.8$. The radial profiles of the dominating spectral line contrast $C(x)$ and frequency $F(x)$ are shown in figure $5(\mathrm{~g})$,(h). It is clearly seen in figure $5(\mathrm{~g})$ that $C(x)$ decreases for $x<6 \mathrm{~cm}$ in accordance with the enhanced level of Landau damping in this region. As for the GAM frequency, its values are lying between estimations made with formula 3 in the same manner as in the previous section 3.3 

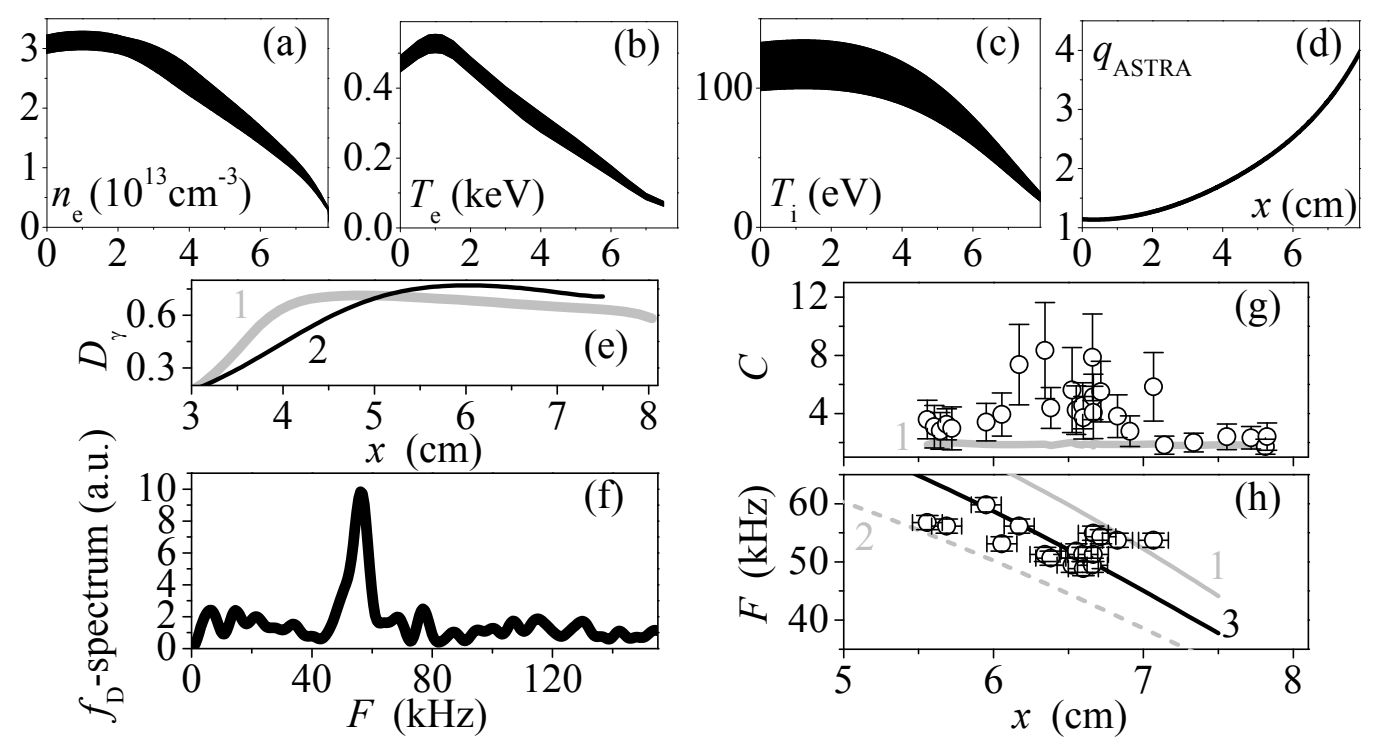

Figure 5. The $32 \mathrm{kA}$ hydrogen regime. Experimental radial profiles of $(a)$ the density; $(b)$ electron and $(c)$ ion temperature. $(d)$ The safety factor profile. (e) GAMs damping curves $(1-19 \mathrm{kA} ; 2-32 \mathrm{kA})$. $(f)$

The $f_{\mathrm{D}}$ spectrum at $x \sim 6.2 \mathrm{~cm}$. Profiles of the dominating line's $(g)$ contrast and $(h)$ frequency.

(look at grey curves 1 and 2 in figure 5(h)). The black curve 3, which was calculated with the formula from ref. [17] for plasmas with two ion species $\left(\mathrm{H}^{+}\right.$and $\mathrm{O}^{8+}$ in this case) falls again between them. The variation of the GAM frequency with radius is smaller in this case, compared to the $19 \mathrm{kA}$ discharge, where it followed the dependence prescribed by the temperature profile. This change of the behavior at growing ohmic power is similar to that observed recently at DIII-D [19] in experiments with intensive additional heating and which was interpreted in terms of the GAM spatial dispersion growing with increasing ion Larmor radii. However, it should be mentioned that this interpretation is probably not applicable, in the case of FT-2, where the ion temperature was comparable for discharges with $19 \mathrm{kA}$ and $32 \mathrm{kA}$ in the region $x=5.5-7 \mathrm{~cm}$ (where GAMs were observable): $T_{\mathrm{i} 19 \mathrm{kA}}=T_{\mathrm{i} 32 \mathrm{kA}}=80 \pm 12 \mathrm{eV}$ at $x=5.5 \mathrm{~cm} ; T_{\mathrm{i} 19 \mathrm{kA}}=53 \pm 8 \mathrm{eV}$ and $T_{\mathrm{i} 32 \mathrm{kA}}=45 \pm 7 \mathrm{eV}$ at $x=7 \mathrm{~cm}$.

\subsection{Simultaneous observation of GAM and MHD mode}

All experiments considered above, are characterized by a low level of magneto hydrodynamic (MHD) activity, which often interferes with GAM measurements with less localized techniques. Accordingly, the MHD mode frequencies, which are typically high in the small FT-2 machine, were not seen in the UHR BS signal and no coherence between the MHD and BS signals was observed. The high locality of the UHR BS gives rise to a fundamentally weak sensitivity to MHD perturbations. In order to demonstrate this, a special current ramp up experiment was performed on FT-2 resulting in a plasma characterized by high MHD activity and a fast variation of the positions of resonant surfaces [20].

The measurements were carried out before and after fast ( $20 \mathrm{MA} / \mathrm{s})$ current ramp ups (CRU) in discharges with $n_{\mathrm{e}}(0)<2.6 \times 10^{19} \mathrm{~m}^{-3}, T_{\mathrm{e}}(0)<510 \mathrm{eV}, T_{\mathrm{i}}(0)<140 \mathrm{eV}, B<2.2 \mathrm{~T}$. The plasma current increases from $22 \mathrm{kA}$ to $32 \mathrm{kA}$ at $t \sim 30 \mathrm{~ms}$ as shown in figure 6(a). The safety profile's evolution reconstructed from ASTRA code simulations taking into account experimental profiles of the density, temperatures and radiation losses is presented in figure $6(\mathrm{~b})$. The radial UHR BS positions for the probing frequency $f_{\mathrm{i}} \sim 65 \mathrm{GHz}$ are shown by 
the dashed black curve in figure 6(b). As shown, the UHR is generally situated between the $q=3$ and $q=2$ surfaces, approaching the later one at $t \sim 31.5 \mathrm{~ms}$. Examples of averaged frequency power spectra of $f_{\mathrm{D}}(t)$ signals measured by quadrature UHR BS technique with $f_{\mathrm{i}} \sim 65 \mathrm{GHz}$ at $t \sim 28 \mathrm{~ms}$ and $t \sim 31.5 \mathrm{~ms}$ (see circles in figure 6(b)) are shown by black curves (labelled as 1) in figure 6(c) and figure 6(e), respectively. The power spectra $A_{\mathrm{MHD}}(t)$ measured by Mirnov coils for the same moments are shown in figure 6(c),(e) by grey curves (labelled as 2). Vertical dashed black lines in these figures indicate GAM frequencies calculated at the local UHR BS positions with the formula from ref. [17]. As it is seen in figure 6(c) the MHD frequency is close to the GAM frequency at $t \sim 28 \mathrm{~ms}$, however it do not coincide with it. The coherence spectrum of the two signals: $f_{\mathrm{D}}(t)$ and $A_{\mathrm{MHD}}(t)$ at $t \sim 28 \mathrm{~ms}$ calculated as

$$
\operatorname{coh}(F)=\frac{\left|\left\langle A_{\mathrm{MHD}}(F) f_{\mathrm{D}}^{*}(F)\right\rangle\right|}{\sqrt{\left\langle A_{\mathrm{MHD}}(F) A_{\mathrm{MHD}}^{*}(F)\right\rangle\left\langle f_{\mathrm{D}}(F) f_{\mathrm{D}}^{*}(F)\right\rangle}}
$$

is shown in figure 6(d) by the black curve 1 . The coherence noise level is plotted by grey line 2. It is obvious that any correlation between these signals is absent. Exactly this case was typical for regimes presented in the previous sections, where the level of the MHD signal was distinctively less.

After the current ramp, at $t \sim 31.5 \mathrm{~ms}$, two intense spectral lines are clearly seen in the $f_{\mathrm{D}}$ spectrum (see figure 6(e)). The dominating one (at $F \sim 64 \mathrm{kHz}$ ) is close to the theoretically predicted GAM frequency, while the second line coincides with the spectral line registered simultaneously by Mirnov coils (at $F \sim 53 \mathrm{kHz}$ ). Phase measurements by two Mirnov coils separated in poloidal direction, allowed to define the poloidal mode number yielding $\mathrm{m}=2$
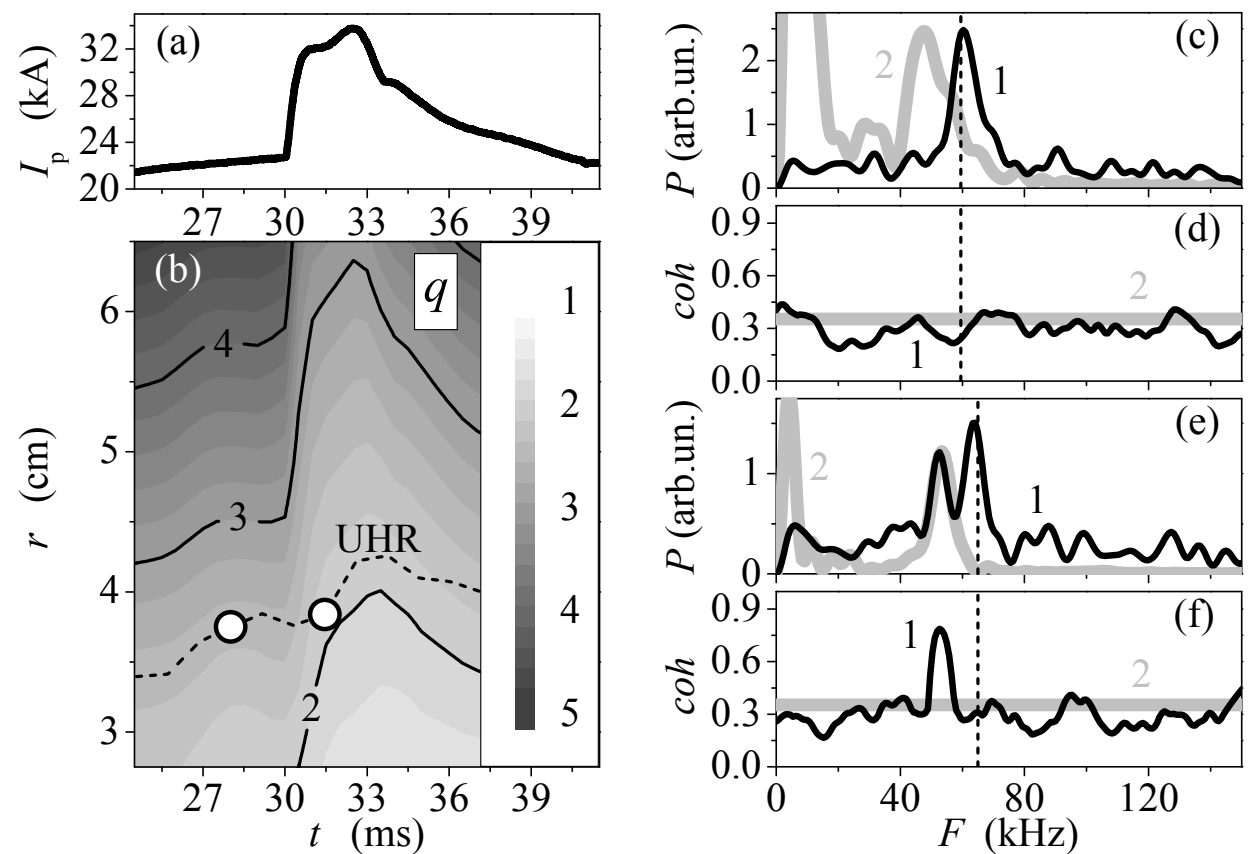

Figure 6. CRU regime. (a) The plasma current's evolution. (b) The safety factor via magnetic surface radius and time (dashed curve - the UHR track at $f_{\mathrm{i}} \sim 65 \mathrm{GHz}$ ). (c) Power spectra measured at $t \sim 28 \mathrm{~ms}$ $\left(1-f_{\mathrm{D}}(t)\right.$ for $f_{\mathrm{i}} \sim 65 \mathrm{GHz} ; 2$ - the MHD signal from Mirnov coils). $(d)$ The coherence spectrum of $f_{\mathrm{D}}$ and MHD signals at $t \sim 28 \mathrm{~ms}$. ( $2-$ the coherence noise level) (e) Power spectra measured at $t \sim 31.5 \mathrm{~ms}(1-$ $f_{\mathrm{D}}(t)$ for $\left.f_{\mathrm{i}} \sim 65 \mathrm{GHz} ; 2-\mathrm{MHD}\right)$. (f) The coherence spectrum of $f_{\mathrm{D}}$ and MHD signals at $t \sim 31.5 \mathrm{~ms}$. 
for the $F=53 \mathrm{kHz}$ mode. The coherence level of the $f_{\mathrm{D}}(t)$ and $A_{\mathrm{MHD}}(t)$ signals at $F \sim 53 \mathrm{kHz}$ is high: $c o h=0.8$ demonstrating the possibility of local MHD mode studies using the Doppler BS diagnostic. The physics reason making the MHD mode visible in the Doppler BS signal is presumably related to the plasma density modulation by the MHD mode and corresponding oscillation of the poloidal component of the probing wave vector (see formula 1), which is perpendicular to the UHR surface (as shown in figure 1(c)). The poloidal projection of the wave vector changes because of distortion of both the magnetic and UHR surfaces by the MHD oscillation. The reason of such a strong influence of the MHD at $t \sim 31.5 \mathrm{~ms}$, most likely, is the proximity of the UHR BS position to the resonant surface $q=2$, as seen in figure 6(b). However, even under these circumstances it was possible to discriminate the MHD contribution to the UHR BS spectrum and analyse the GAM properties.

\section{The spatial structure of GAMs}

\subsection{Correlation studies with UHR Doppler BS and $\boldsymbol{O}$-mode reflectometry}

The radial spatial structure of GAMs in FT-2 tokamak was investigated in the $19 \mathrm{kA}$ hydrogen discharge (introduced in the section 3.1) utilising two simultaneously measured signals for a correlation analysis. The reference signal is the homodyne detected signal $A_{\mathrm{rfl}}(t)$ of the $O$ mode reflectometer (shifted by 90 degrees toroidally with respect to the UHR BS location) with top launching and almost vertical probing sensitive to the density fluctuations near the cutoff layer. The appropriate ray trajectory at probing frequency $f_{\mathrm{i}} \sim 27.7 \mathrm{GHz}$ is shown in figure 7(a) by the solid black curve. The power spectrum of this signal calculated in the time interval $\Delta t \sim 4.6 \mathrm{~ms}$ (with 112 averaging windows taken one by one) is shown above in figure $3(\mathrm{e})$ by the black curve 1 . The dominating line at the GAM frequency $F_{\mathrm{G}} \sim 44 \mathrm{kHz}$ is clearly observed.

The second signal used in the correlation analysis is the UHR BS Doppler frequency shift evolution $f_{\mathrm{D}}(t)$, reconstructed with the help of the signals of the quadrature UHR BS scheme both measured simultaneously with the reference reflectometric signal. The UHR BS probing frequency was changed in steps $\Delta f_{\mathrm{i}}=1 \mathrm{GHz}$ in the range $f_{\mathrm{i}} \sim 55-68 \mathrm{GHz}$ corresponding to the UHR BS positions plotted by circles in figure 7(a). Each position was calculated as the intersection of the $X$-mode ray trajectory (central one in the beam), started parallel to the major radius from the high field side $(x=-8.1 \mathrm{~cm}, y=1.5 \mathrm{~mm})$, and the appropriate UHR surface. Small segments of these UHR surfaces are shown as grey solid segments in figure 7(a). These segments are limited by the level of the BS signal dropping below $-3 \mathrm{~dB}$ of the respective maximal value indicated by the circle. Circular flux surfaces passing through the data points are shown by light grey dashed curves. One of them, the nearest to the turning point of the reflectometric ray trajectory, corresponds to the UHR BS position calculated for $f_{\mathrm{i}} \sim 63.2 \mathrm{GHz}$. The spectrum analysis of the $f_{\mathrm{D}}(t)$ signal was presented in detail in section 3.3. Keeping in mind the GAM symmetry $\left(\mathrm{m}=0\right.$ for $E_{r}$ and $\mathrm{m}=1$ for density $\left.n\right)$, it is obvious to explore the coherence of two signals in terms of the distance between magnetic surfaces where BS occurs and where the reflectometry turning point is situated. In this section we calculate the distance along the $y$ axes, fixing the $\Delta y=0$ at the reflectometric turn point $(r \sim 5 \mathrm{~cm})$. The conversion of this distance to the flux surface radii difference could be also easily done using the ratio $\Delta y / \Delta r \sim 0.95$.

The cross-correlation function (CCF) of $A_{\mathrm{rff}}(t)$ and $f_{\mathrm{D}}(t)$ signals was calculated for each frequency component $F$ as: 

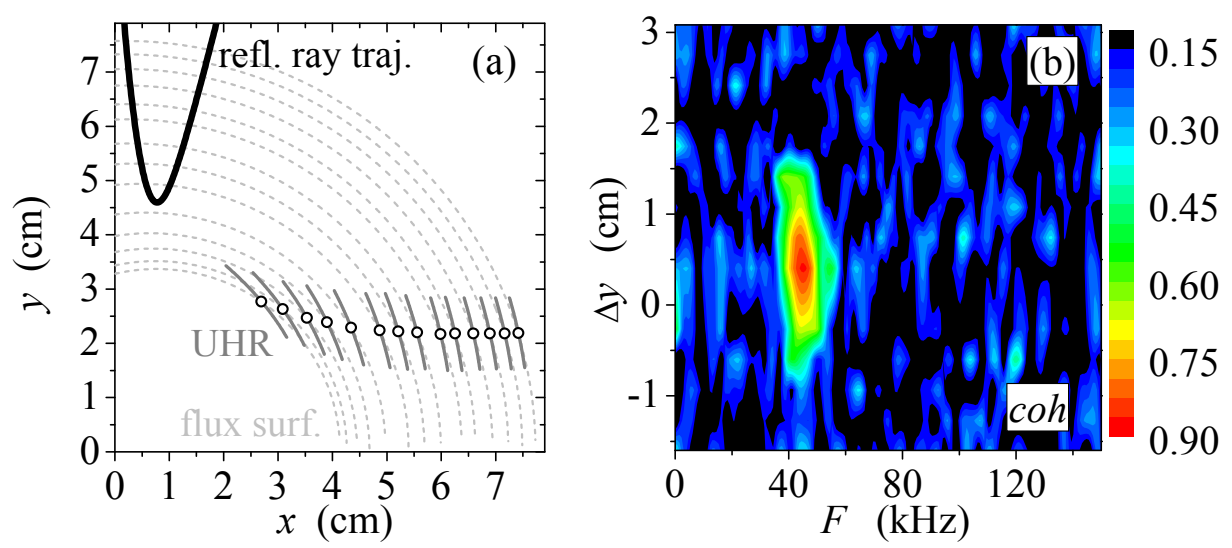

Figure 7. (a) The correlation experiment's geometry. (b) The coherence spectrum of the reflectometric $A_{\text {rfl }}$ and the UHR BS $f_{\mathrm{D}}$ signals (the mean noise level corresponds to $c o h \sim 0.14$ ). (Colours adapted to grey scale in figure $(b)$ in the printed version of the article.)

$$
K(F, \Delta y)=\left\langle A_{\mathrm{rfl}}(F) f_{\mathrm{D}}^{*}(F, \Delta y)\right\rangle .
$$

The coherence spectrum of the UHR BS $f_{\mathrm{D}}$-dependence and $O$-mode reflectometer signal calculated as:

$$
\operatorname{coh}(F, \Delta y)=\frac{|K(F, \Delta y)|}{\sqrt{\left\langle A_{\mathrm{rfl}}(F) A_{\mathrm{rfl}}^{*}(F)\right\rangle\left\langle f_{\mathrm{D}}(F, \Delta y) f_{\mathrm{D}}^{*}(F, \Delta y)\right\rangle}}
$$

is shown in figure 7(b). It is important to stress the high level of the coherence, clearly observable at the GAM frequency where coh $\sim 0.9$, which confirms the feasibility of this correlation approach on one hand. On the other, this result is an additional argument in favour of the GAM origin of the Doppler UHR BS and reflectometer signals, because they were found to be globally correlated. The area of high coherence at $F \sim F_{\mathrm{G}}$ is elongated along the $\Delta y$ axis. The coherence maximum is not located at the $\Delta y=0$ point, rather shifted from the cutoff position by $0.4-0.5 \mathrm{~cm}$ outwards, which is probably due to the reflectometer localisation properties. The coherence drops by a factor of $e$ at $\Delta y=+1.6 \pm 0.2 \mathrm{~cm}$ and $\Delta y=-$ $0.8 \pm 0.2 \mathrm{~cm}$. The value of the error here corresponds to the frequency step (associated with the respective spatial displacement) in the second UHR BS channel and does not take into account the spatial locality of the reference reflectometric channel. The resulting GAM radial correlation length can be estimated as $l_{\mathrm{c}} \sim 1.3 \pm 0.2 \mathrm{~cm}$.

It is worth to note, that the dominating lines in the $f_{\mathrm{D}}(t)$ and reflectometer spectra are associated with different GAM components: $V_{\theta}\left(\right.$ or $E_{r}$ ) and $n$, respectively. The reflectometric and UHR BS channels were operating in different poloidal cross-sections, shifted toroidally by 90 degrees. Nevertheless the high level of coherence was observed in the experiment at the GAM frequency, which is evident for a global mode. Moreover, the cross-phase of $A_{\mathrm{rfl}}(t)$ and $f_{\mathrm{D}}(t)$ signals at the GAM frequency calculated in the coherency maximum position at $\Delta y \sim 0.45 \mathrm{~cm}$ is close to $\pi / 2$, which is in agreement with the GAM theory predictions for $E_{\mathrm{r}}$ and density components. 


\subsection{GAM studies with the dual-frequency correlative UHR BS scheme}

Implementation of the reflectometer in the correlative scheme restricts the measurement spatial resolution. The alternative scheme, utilising two UHR BS channels, was developed to improve it up to the sub-mm level.

In order to simplify the measurement scheme two UHR BS signals at probing frequencies $f_{1}$ and $f_{2}$, corresponding to probing diagram central ray UHR spatial separation $\Delta L$ in the plasma poloidal cross-section, are measured simultaneously by two homodyne channels. In general, the peculiarity of the homodyne spectrum is an overlapping of the spectral components with the same turbulence frequencies but with opposite phase velocities. Because of the large Doppler frequency shift of the quadrature spectrum (see figure 2(i)) the overlapping effect is, however, small enough to ensure reliable determination of the variable Doppler frequency shift based on homodyne spectra. Therefore, using the homodyne spectra two oscillating signals $f_{\mathrm{D} 1}(t)$ and $f_{\mathrm{D} 2}(t)$ can be reconstructed in the same manner as it was described in section 3.2 for the quadrature spectrum (see formula 2). Due to the modulation of both signals $\left(f_{\mathrm{D} 1}\right.$ and $\left.f_{\mathrm{D} 2}\right)$ a high level of correlation between them can be expected just at the GAM frequency.

The corresponding measurements were performed in the $19 \mathrm{kA}$ hydrogen discharge (see the parameters in sections 3.1 and 4.1) with the correlative scheme described in [7]. This scheme was adopted for the frequency difference up to $\left|f_{2}-f_{1}\right|=4 \mathrm{GHz}$, corresponding to a spatial separation up to $|\Delta L| \sim 2 \mathrm{~cm}$, required for the meso-scale structure investigation. The sub-mm spatial resolution of the UHR BS allows to resolve fine details in the mode structure when small enough $\left(\left|\delta f_{2}\right| \cong 20 \mathrm{MHz}\right)$ frequency steps are used in measurements. As a result the Doppler frequency shift temporal variation in both channels is determined and their crosscorrelation spectra

$$
K(F, \Delta L)=\left\langle f_{\mathrm{D} 1}(F) f_{\mathrm{D} 2}^{*}(F, \Delta L)\right\rangle
$$

are calculated. The coherence spectrum defined as

$$
\operatorname{coh}(F, \Delta L)=\frac{|K(F, \Delta L)|}{\sqrt{\left\langle f_{\mathrm{D} 1}(F) f_{\mathrm{D} 1}^{*}(F)\right\rangle\left\langle f_{\mathrm{D} 2}(F, \Delta L) f_{\mathrm{D} 2}^{*}(F, \Delta L)\right\rangle}}
$$

is shown in figure 8 (a) for the reference frequency $f_{1}=64.1 \mathrm{GHz}$. The spatial separation $\Delta L$ was calculated as the distance between central BS points for each pair of probing beams. For the subsequent comparison of results of correlation measurements it is convenient to use instead of the distance $\Delta L$ in the radial-poloidal plane the spatial separation $\Delta r$ between magnetic surfaces through-passing the same BS points. The conversion to the flux surface radii difference could be done in the particular experiment using relation $\Delta L / \Delta r \sim 1.2 \pm 0.02$. The maximum of the coherence coh $\sim 0.91$ corresponds to the frequency $F \sim F_{\mathrm{G}}$ at $\Delta L \sim 0$ $(r \sim 4.3 \mathrm{~cm})$ and the coherence drops by a factor of $e$ for $\Delta L=+0.72 \pm 0.01 \mathrm{~cm}$ and $\Delta L=-$ $0.94 \pm 0.01 \mathrm{~cm}$. The elongation of the high coherence area at negative $\Delta L$ could be explained by the degradation of the spatial (in minor radii) resolution due to the growth of the angle between the UHR and magnetic surfaces, which indicated in figure 7(a), where the UHR surface fragments intersect more flux surfaces in the inner region. The resulting radial correlation length is given by $l_{\mathrm{c}} \sim 0.7 \pm 0.02 \mathrm{~cm}$, which is a factor of 2 smaller than the estimation made in section 4.1. This difference is probably explained by the poor spatial resolution of the reflectometry diagnostics; part of it can also be attributed to a slightly different position of the measurement point: the reflectometer turning point is shifted 

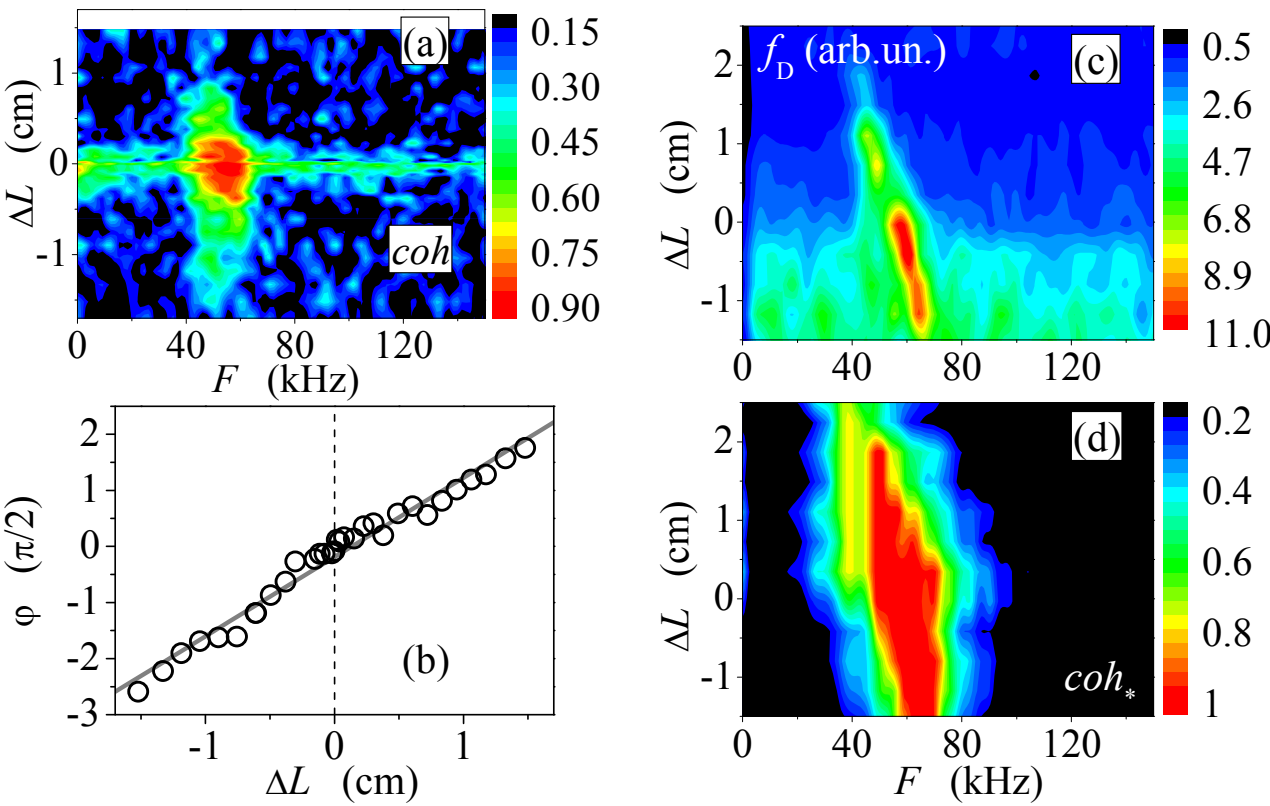

Figure 8. (a) The coherence spectrum of two $f_{\mathrm{D}}$ signals reconstructed from two homodyne UHR BS spectra (the mean noise: coh 0.14). (b) The cross-phase dependence via $\Delta y$ at $F \sim 46 \mathrm{kHz}$. (c) The power spectrum of the $f_{\mathrm{D}}(t)$ signal via $\Delta L$. (d) Simulation of the coherence spectrum. (Colours adapted to grey scale in figures (a), (c) and (d) in the printed version of the article.)

outwards in radius by $\sim 0.7 \mathrm{~cm}$ with respect to the Doppler BS reference channel's UHR radial position.

The cross-phase spectrum of the two Doppler UHR BS signals $f_{\mathrm{D} 1}(t)$ and $f_{\mathrm{D} 2}(t)$, calculated from the ratio of imaginary and real parts of the CCF (formula 9) as follows:

$$
\varphi(F, \Delta L)=\operatorname{arctg}\left[\frac{\operatorname{Im}(K(F, \Delta L))}{\operatorname{Re}(K(F, \Delta L))}\right],
$$

is plotted in figure $8(\mathrm{~b})$ for $F=F_{\mathrm{G}}$. As it is seen, the $\varphi(\Delta L)$ dependence is linear in a wide range larger than $2 \pi$. This dependence allows us to determine the GAM wave number as $k_{L}=d \varphi / d \Delta L=2.2 \mathrm{~cm}^{-1}\left(k_{r} \sim 2.6 \mathrm{~cm}^{-1}\right)$ and the GAM radial phase velocity $V_{r}=1.2 \pm 0.1 \mathrm{~km} / \mathrm{s}$ directed outwards, to the direction of decreasing electron temperature, as often observed in experiment [2]. This observation is in qualitative agreement with theory [21] accounting for GAM spatial dispersion and predicting its localization at temperatures smaller than that satisfying for a given frequency relation (3). However it should be stressed that the Airy function standing wave structure, predicted by theory $[21,22]$ is not consistent with observation of running wave-pattern made at JFT-2M [3], HL-2A [4], TEXTOR [6, 23], DIIID [19] and now at FT-2. This inconsistency is appealing for further theoretical and experimental investigations clarifying the physical reason for GAM wave number direction and value. The GAM radial wavelength $(2.4 \mathrm{~cm})$ in FT-2 appears to be substantially larger than its correlation length $(0.7 \mathrm{~cm})$, which is similar to the findings obtained at TEXTOR [23] but differs from opposite observations made recently at TCV [24].

The mechanism of such a strong GAM radial coherence suppression is undoubtedly of great interest. A simple explanation is given by assumption that the coherence suppression is related to the decreasing overlap of GAM spectral lines in the two signals at growing separation $\Delta L$ due to the radial dependence of the GAM frequency as shown in figure 8(c). To check this hypothesis we simulated the coherence spectrum as follows: 


$$
\operatorname{coh}_{*}(F, \Delta L)=\sqrt{\frac{P_{\text {fit }}(F) P_{\text {fit }}(F, \Delta L)}{P(F) P(F, \Delta L)}},
$$

where $P(F)$ is the experimental $f_{\mathrm{D}}$-spectrum measured by quadrature scheme at the probing frequency $f_{\mathrm{i}}=64.1 \mathrm{GHz}$ corresponding to the reference channel in the correlation scheme $(\Delta L=0) ; P(F, \Delta L)$ is the experimental $f_{\mathrm{D}}$-spectrum measured in the channel at a distance $\Delta L$ from the first one; $P_{\text {fit }}$ is the Lorenz like fit of the line in the $f_{\mathrm{D}}$-spectrum possessing the central frequency $F_{\mathrm{G}}$ depending on $\Delta L$. It is necessary to explain that the experimental spectra in the denominator contains both GAM line and noise, whereas Lorenzian GAM spectrum fits in the numerator don't contain noise components. Therefore in this model the coherence should decrease proportionally to $\sqrt{\frac{P_{\mathrm{fit}}(F, \Delta L)}{P(F, \Delta L)}}$ (square root of the signal-to-noise ratio) at growing distance $\Delta L$. The coherence calculated with formula (12) is presented in figure $8(\mathrm{~d})$. It is clearly seen in figures 8 (a),(d) that the experimental radial correlation length is much smaller than that prescribed by formula (12). Accordingly the small GAM radial correlation length in FT-2 is most likely not related to the GAM frequency radial dependence, rather the opposite statement could be true: the GAM frequency can radially vary due to its small correlation length. The later statement is indirectly supported by the fact that no dependence of the GAM frequency on radius was observed at TCV where large correlation length is reported [24].

\section{Interaction of GAMs with high frequency drift turbulence}

According to the widely accepted paradigm, GAMs are driven in tokamaks due to Reynolds stress provided by small-scale drift-wave turbulence. In theoretical terms it happens due to the three-wave interaction of the background drift-wave fluctuations with this global mode, which occurs over the GAM localization region. On its turn the GAMs - excited to a substantial level - can shear drift-wave eddies leading to suppression of long-scale fluctuations. They also couple drift wave fluctuations as a result of three-wave interactions, thus influencing the turbulence radial wave number spectrum formation at small scales [25]. The evidence for the later local three-wave process is manifested, in principal, in the phases of the interacting wave. This information is usually [2] extracted from the experimental data applying the bicoherency analysis [26].

In this section we apply the Doppler UHR BS diagnostics, being well localized in radial direction, to the visualization of GAM - turbulence interaction.

The auto-bicoherence spectrum of the UHR BS signal proportional to the phase factors of small-scale fluctuations contributing to it revealed an absence of the non-linear coupling between different small-scale turbulence components. However this negative result does not prove the absence of GAM - turbulence three-wave-interaction because the long-scale GAM wave is not contributing to the UHR BS directly, but is rather leading to the BS signal frequency modulation due to plasma poloidal velocity oscillations at the GAM frequency. Under these circumstances the cross-bicoherence spectra can be useful for studying the broadband turbulence control by GAMs due to three-wave interaction. The spectra are given by the following expressions:

$$
b_{1}^{2}\left(f_{1}, f_{2}\right)=\frac{\left|\left\langle A_{\mathrm{UHR}}\left(f_{1}\right) A_{\mathrm{UHR}}\left(f_{2}\right) f_{\mathrm{D}}^{*}\left(f_{1} \pm f_{2}\right)\right\rangle\right|^{2}}{\left\langle\left|A_{\mathrm{UHR}}\left(f_{1}\right) A_{\mathrm{UHR}}\left(f_{2}\right)\right|^{2}\right\rangle\left\langle\left|f_{\mathrm{D}}\left(f_{1} \pm f_{2}\right)\right|^{2}\right\rangle},
$$




$$
b_{2}^{2}\left(f_{1}, f_{2}\right)=\frac{\left|\left\langle A_{\mathrm{UHR}}\left(f_{1}\right) f_{\mathrm{D}}\left(f_{2}\right) A_{\mathrm{UHR}}^{*}\left(f_{1} \pm f_{2}\right)\right\rangle\right|^{2}}{\left\langle\left|A_{\mathrm{UHR}}\left(f_{1}\right) f_{\mathrm{D}}\left(f_{2}\right)\right|^{2}\right\rangle\left\langle\left|A_{\mathrm{UHR}}\left(f_{1} \pm f_{2}\right)\right|^{2}\right\rangle},
$$

where $A_{\mathrm{UHR}}\left(f_{1}\right), A_{\mathrm{UHR}}\left(f_{2}\right)$ and $A_{\mathrm{UHR}}\left(f_{1} \pm f_{2}\right)$ are different harmonics of the complex Fourier spectrum of the UHR BS signal (one of two sine-cosine homodyne detected signals), which are proportional to density fluctuations at the same frequency $[7,8], f_{\mathrm{D}}\left(f_{2}\right)$ and $f_{\mathrm{D}}\left(f_{1} \pm f_{2}\right)$ are harmonics of the Doppler frequency shift signal spectrum extracted from the UHR BS signal using procedures described above.

The level of $b^{2}$ will exceed the noise level if the phase of the turbulence spectral components (associated with the UHR BS signal) are coupled with the phase of the Doppler frequency shift $f_{\mathrm{D}}$ under the conditions of three-wave interaction. The $f_{\mathrm{D}}$-signal has a dominant clear spectral line at relatively low GAM-frequency $f_{2} \sim F_{\mathrm{G}}=(30-60) \mathrm{kHz}$, which determines a relatively long sampling time needed for proper frequency resolution. Provided a limited duration of the discharge flat-top, the later condition impose a limitation on bicoherence spectrum calculations with sufficient level of averaging.

An example of the cross-bicoherence spectrum $b_{1}^{2}$ calculated by formula 13 for $19 \mathrm{kA}$ hydrogen discharge is shown in figure 9 (a) and its low and high frequency parts are presented in figure 9(b, c). The sampling rate for initial IQ signals was $50 \mathrm{MHz}$. The evolution of the quadrature FFT power spectrum was calculated for single windows with 128 points with a step on 4 points, which provided the reconstruction of the $f_{\mathrm{D}}$-signal with a sampling rate 12.5 MHz. This signal was used for calculation of the cross-bicoherence spectrum by formula 13/14 together with IQ signals re-sampled down to the same rate $12.5 \mathrm{MHz}$. The window with 512 data points was used in one FFT realization. Each next window was started after the end of the previous one (512 points for step). The averaged bicoherence spectrum was calculated over 256 realizations. As it is clearly seen in figure $9(a-c)$ the level of

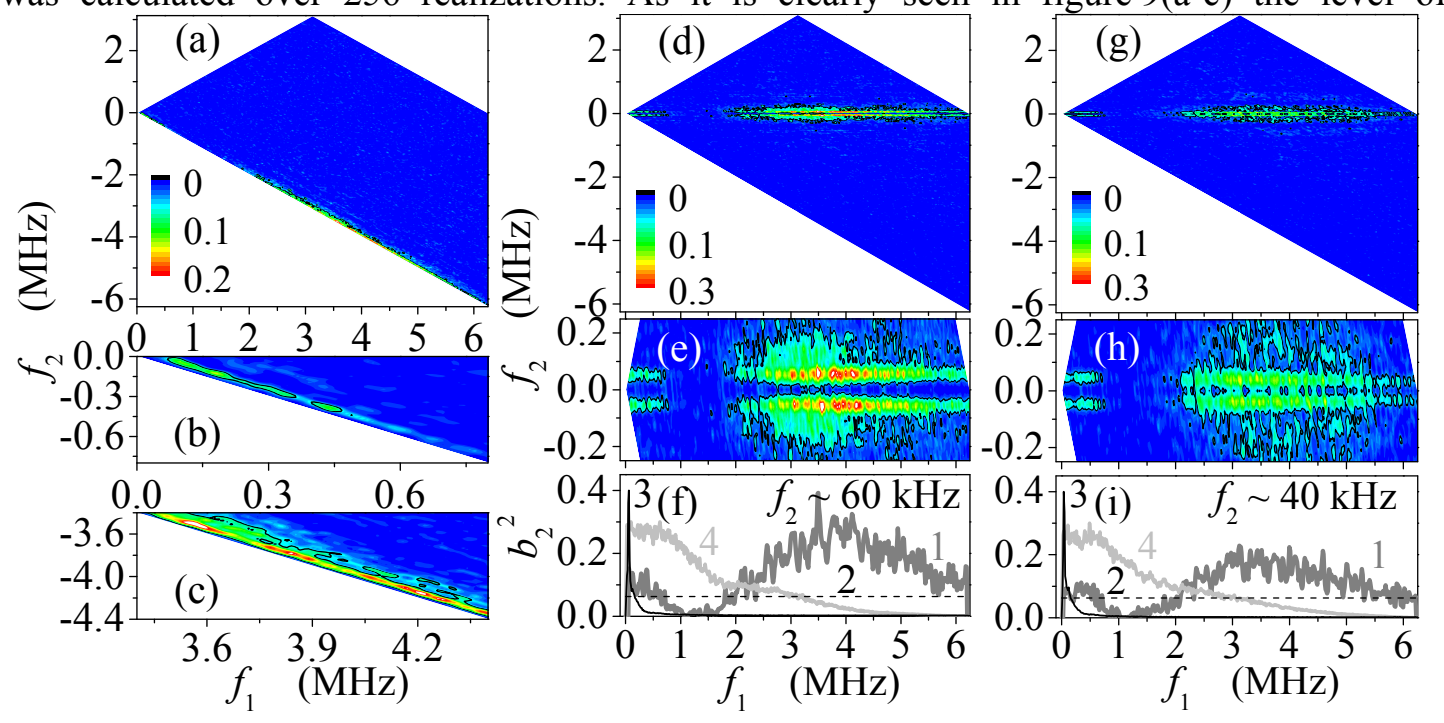

Figure 9. The $19 \mathrm{kA}$ hydrogen case: (a) cross-bicoherence spectrum $b_{1}^{2} ;(b)$ its low and (c) high frequency zooms (black contours: the noise level $\left.b_{\mathrm{n}}{ }^{2}\right) ;(d)$ cross-bicoherence spectrum $b_{2}{ }^{2} ;(e)$ its low frequency zoom; $(f) b_{2}^{2}\left(f_{1}\right)$ dependence at $f_{2} \sim 60 \mathrm{kHz}(1), b^{2}$-noise $(2), f_{\mathrm{D}}(3)$ and $A_{\mathrm{UHR}}$ (4) spectra. The 19 kA deuterium case: $(g)$ cross-bicoherence spectrum $b_{2}{ }^{2} ;(h)$ its low frequency zoom; $(i) b_{2}{ }^{2}\left(f_{1}\right)$ at $f_{2} \sim 40 \mathrm{kHz}(1), b^{2}$-noise (2), $f_{\mathrm{D}}(3)$ and $A_{\mathrm{UHR}}(4)$ spectra. (Colours adapted to grey scale in figures (a-e), ( $\mathrm{g}-\mathrm{h})$ in the printed version of the article.) 
$b_{1}^{2}\left(f_{1}, f_{2}\right)$ exceeds the noise level $b_{\mathrm{n}}{ }^{2} \sim 0.06$ at $f_{2}=F_{\mathrm{G}}-f_{1}$ (where $F_{\mathrm{G}} \sim 60 \mathrm{kHz}$ ) in two wide frequency ranges: $f_{1 / 2}<700 \mathrm{kHz}$ and $f_{1 / 2}>2 \mathrm{MHz}$, demonstrating the strong nonlinear threewave interaction between small-scale turbulence and GAM. In the low-frequency domain $\left(f_{1 / 2}<700 \mathrm{kHz}\right)$ where the turbulence amplitude is high and radial wave number is relatively small $[7,8]$ one can expect generation of GAMs by the Reynolds stress via the three-wave coupling. On contrary, at high frequency of turbulent fluctuations $\left(f_{1 / 2}>2 \mathrm{MHz}\right)$ and radial wave number their amplitude is small $[7,8]$ and Reynolds stress should be negligible. In the later case rather intensive GAMs are providing drift fluctuation coupling due to the threewave interaction thus participating in the turbulence spectrum formation [25].

An example of the cross-bicoherence spectrum $b_{2}^{2}$ calculated by formula 14 for the same case is shown in figure 9(d) and its low frequency part is presented in figure 9(e). The level of $b_{2}^{2}\left(f_{1}, f_{2}\right)$ exceeds the noise level $b_{\mathrm{n}}$ at $f_{2}= \pm F_{\mathrm{G}} \sim \pm 60 \mathrm{kHz}$ in two frequency ranges: $f_{1}<700 \mathrm{kHz}$ and $f_{1}>2 \mathrm{MHz}$. The maximal value of $b_{2}^{2}$ is close to $30 \%$. This result as well clearly demonstrates the existence of the nonlinear mechanism of the small-scale turbulence spectrum formation assisted by GAMs [25] both at low and high frequencies. The dependence of $b_{2}^{2}\left(f_{1}\right)$ obtained at $f_{2}=60 \mathrm{kHz}$ is shown by grey curve 1 in figure $9(\mathrm{f})$, together with the noise level (dashed black curve 2) and with two power spectra of $f_{\mathrm{D}}$ (continuous black curve 3 ) and $A_{\text {UHR }}$ (light grey curve 4 ) signals, both presented in arbitrary units.

The cross-bicoherence and power spectra for the $19 \mathrm{kA}$ deuterium discharge are presented in figure 9(g-i). The frequency resolution for bicoherence calculation was $\Delta f_{1 / 2} \sim \pm 6 \mathrm{kHz}$, which was found sufficient to allow detecting the small shift of the GAM frequency down to $f_{2}= \pm F_{\mathrm{G}} \sim \pm 40 \mathrm{kHz}$ in the deuterium case. The value of $b_{2}^{2}$ in this case is lower than in hydrogen, and its maximal level is close to $20 \%$. The high frequency range where the nonlinear interactions are observed because of that looks narrower in deuterium case.

Summarizing this section we conclude that bicoherence analysis of the UHR BS signals is sufficiently local and sensitive to detect the nonlinear three-wave processes, according to [25], forming the turbulence small-scale component wave number spectra in the GAM presence. The method presented here combined with energy confinement analysis looks promising for investigating the GAM - small-scale turbulence interaction and its influence on anomalous transport.

\section{Conclusions}

In summary, on FT-2 tokamak we have shown that the application of highly localised Doppler UHR BS diagnostics to the investigation of geodesic acoustic modes has resulted in a comprehensive characterisation of this global oscillation. The GAM was shown to be excited up to a substantial level in the outer half of low current discharges. However its localisation region was shown to shrink and move towards the edge with growing plasma current (lower q). The global character of the mode was confirmed by the observation of its density component with $m=1, n=0$ symmetry and by its phase shift with respect to the GAM electric field. The GAM frequency dependence on electron temperature was recovered by radial scan, whereas the ion mass dependence was checked in comparative experiments in hydrogen and deuterium. The dependence of the GAM frequency on impurity content as recently predicted by theory gives rise to a reasonable agreement with the experimental observations. Application of the dual frequency measurements accompanied by correlation analysis allowed determining both GAM wavelength and correlation length. These results 
indicate a strong plasma poloidal rotation inhomogeneity introduced by GAMs. Evidence for a GAM interaction with plasma turbulence via three-wave coupling is obtained. This new diagnostic technique, whose potential was demonstrated in this paper, opens further options for studying GAM-turbulence interplay.

\section{Acknowledgments}

Financial support of RFBR grants 13-02-00614 and 10-02-00631, the Russian Academy Presidium program №12, the RF Government grant 11.G34.31.0041, NWO-RFBR Centre-ofExcellence grant 047.018.002 is acknowledged. Members of SPbSPU Research Laboratory of Physics of Advanced Tokamaks (RLPAT) are warmly acknowledged for discussions at seminars.

\section{References}

[1] Diamond P H, Itoh S-I, Itoh K, and Hahm T S 2005 Plasma Phys. Control. Fusion 47 R35

[2] Fujisawa A 2009 Nucl. Fusion 49013001

[3] Ido T et al 2006 Plasma Phys. Control. Fusion 48 S41

[4] Lan T et al 2008 Plasma Phys. Control. Fusion 50045002

[5] Hidalgo C et al 2009 Europhys. Lett. 8755002

[6] Xu Y and et al 2011 Plasma Phys. Control. Fusion 53095015

[7] Gusakov E Z, Gurchenko A D, Altukhov A B, Stepanov A Yu, Esipov L A, Kantor A Yu and Kouprienko D V 2006 Plasma Phys. Control. Fusion 48 A371

[8] Gusakov E Z, Gurchenko A D, Altukhov A B, Bulanin V V, Esipov L A, Kantor A Yu, Kouprienko D V, Lashkul S I, Petrov A V and Stepanov A Yu 2006 Plasma Phys. Control. Fusion 48 B443

[9] Horton W 1999 Rev. Mod. Phys. 71735

[10] Leerink S et al 2012 Phys. Rev. Lett. 109165001

[11] Budnikov V N, Gerasimenko G V, Esipov L A, Dyachenko V V, Levitskii A N, Obukhov A A, Pavlov I P, Stepanov A Yu, Sakharov I E, Shatalin S V and Scherbinin O N 1984 Sov. J. Plasma Phys. 10281

[12] Conway G D et al 2005 Plasma Phys. Control. Fusion 471165

[13] Altukhov A B, Gurchenko A D, Gusakov E Z, Esipov L A, Selenin V L and Stepanov A Yu 2003 Proc. 30 th EPS Conf. on Controlled Fusion and Plasma Physics (St Petersburg, Russia) vol 27A (ECA) P-4.170pd

[14] Leerink S et al, 2012 Proc. 39th EPS Conf. \& $16^{\text {th }}$ Int. Congress on Plasma Physics (Stockholm, Sweden) vol 36F (ECA), P-4.003

[15] Candy J and Waltz R E 2003 J. Comp. Phys. 186545

[16] Hinton F L and Rosenbluth M N 1999 Plasma Phys. Control. Fusion 41 A653

[17] Guo W, Wang S and Li J 2010 Phys. Plasmas 17112510

[18] Gao Z, Itoh K, Sanuki H and Dong J Q 2006 Phys. Plasmas 13100702

[19] Hillesheim J.C., Peebles W.A., Carter T.A., Schmitz L. and Rhodes T.L. 2012 Phys. Plasmas 19022301

[20] Gurchenko A D, Gusakov E Z, Altukhov A B, Stepanov A Yu, Kouprienko D V, Kantor M Yu, Esipov L A and Lashkul S I 2009 Proc. 36th EPS Conf. on Plasma Physics (Sofia, Bulgaria) vol 33E (ECA) P-5.207

[21] Zonca F, Chen L 2008 EPL 8335001

[22] Itoh K, Itoh S-I, Diamond P H, Fujisawa A, Yagi M, Watari T, Nagashima Y and Fukuyama A 2006 Plasma Fusion Res. 1037

[23] Xu Y et al 2011 Proc. 38th EPS Conf. on Plasma Physics (Strasbourg, France) vol 35G (ECA) O4.125

[24] De Meijere C A 2012 PhD thesis CRPP EPFL

[25] Gürcan Ö D, Hennequin P, Vermare L, Garbet X and Diamond P H 2010 Plasma Phys. Control. Fusion 52045002

[26] Kim Y C and Powers E J 1979 IEEE Trans. Plasma Sci. PS-7 120 